Swarthmore College

Works

$11-1-1992$

\title{
Fragment Rotational State Distributions From The Dissociation Of NelBr: Experimental And Theoretical Results
}

Thomas Alex Stephenson

Swarthmore College, tstephe1@swarthmore.edu

Follow this and additional works at: https://works.swarthmore.edu/fac-chemistry

Part of the Physical Chemistry Commons

Let us know how access to these works benefits you

\section{Recommended Citation}

Thomas Alex Stephenson. (1992). "Fragment Rotational State Distributions From The Dissociation Of NelBr: Experimental And Theoretical Results". Journal Of Chemical Physics. Volume 97, Issue 9.

6262-6275. DOI: 10.1063/1.463688

https://works.swarthmore.edu/fac-chemistry/1

This work is brought to you for free by Swarthmore College Libraries' Works. It has been accepted for inclusion in Chemistry \& Biochemistry Faculty Works by an authorized administrator of Works. For more information, please contact myworks@swarthmore.edu. 


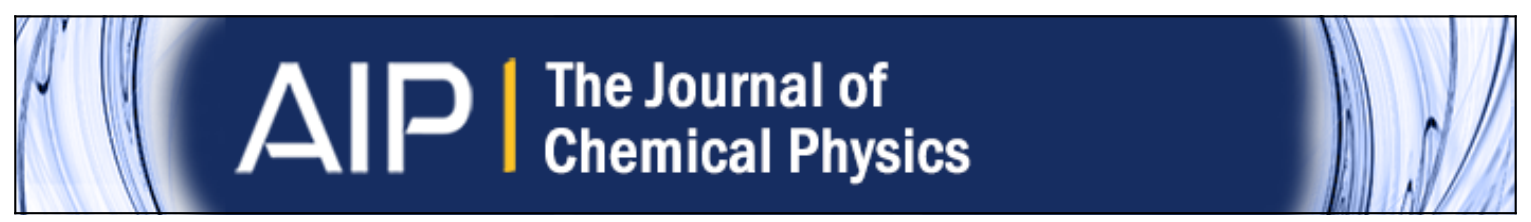

Fragment rotational state distributions from the dissociation of $\mathrm{NelBr}$ :

Experimental and theoretical results

Thomas A. Stephenson

Citation: The Journal of Chemical Physics 97, 6262 (1992); doi: 10.1063/1.463688

View online: http://dx.doi.org/10.1063/1.463688

View Table of Contents: http://scitation.aip.org/content/aip/journal/jcp/97/9?ver=pdfcov

Published by the AIP Publishing

AlIP Re-register for Table of Content Alerts

Create a profile.

Sign up today! 


\title{
Fragment rotational state distributions from the dissociation of NelBr: Experimental and theoretical results
}

\author{
Thomas A. Stephenson \\ Department of Chemistry, Swarthmore College, Swarthmore, Pennsylvania 19081
}

(Received 28 May 1992; accepted 13 July 1992)

\begin{abstract}
The IBr fragment rotational state distributions that result when the $\mathrm{NeIBr}$ van der Waals molecule undergoes vibrational predissociation have been measured in a pump-probe laser-induced fluorescence experiment. Independent of initial vibrational state and the number of quanta of vibrational energy lost from the $\mathrm{I}-\mathrm{Br}$ coordinate, the rotational distributions extend over the full range of energetically accessible states. From the observation of energetic constraints on the rotational distribution, the dissociation energy $\left(D_{0}\right)$ is calculated to be 65.5 $\pm 1.2 \mathrm{~cm}^{-1}$ for the $A$ electronic state, $v=16$. For the $X$ electronic state, $v=0, D_{0}=71.8 \pm 1.2$ $\mathrm{cm}^{-1}$. Quantum mechanical bound state calculations carried out on a model $A$ electronic state potential energy surface are in quantitative agreement with this result. The rotational distributions are broader than that predicted by either a Franck-Condon or classical impulsive model for the dissociation. The distributions are qualitatively in accord with classical trajectory calculations. Analysis of specific rotational distributions in the context of the energy gap law shows poor quantitative agreement. The average fragment rotational energy relative to analogous Ne-containing complexes is, however, qualitatively predicted by the energy gap relations.
\end{abstract}

\section{INTRODUCTION}

The structure and vibrational predissociation dynamics of rare gas/halogen van der Waals molecules have proved to be an important probe of atom-diatom long range interaction potentials. ${ }^{1}$ The result of over 15 years of active research in this field has been a large database of equilibrium structures, binding energies, vibrational predissociation lifetimes, and fragment quantum state distributions. ${ }^{1}$ Very early in these studies it became clear that rotational effects were of paramount importance to an understanding of the dynamics of van der Waals complexes. Beswick and Delgado-Barrio demonstrated, for example, that the details of the fragment $I_{2}$ rotational distribution resulting from the dissociation of $\mathrm{HeI}_{2}$ were highly sensitive to the assumed $\mathrm{He}-\mathrm{I}_{2}$ interaction potential. ${ }^{2}$ This result arises despite the presumed minor role that the rotational degree of freedom plays in the dissociation of $\mathrm{HeI}_{2}$ due to the strong kinematic constraints on angular momentum transfer in light atom-heavy diatom combinations. Halberstadt et al. have similarly demonstrated the impact of subtle changes in the $\mathrm{Ne}-\mathrm{Cl}_{2}$ interaction potential on the fragment $\mathrm{Cl}_{2}$ rotational distributions. ${ }^{3}$ Most recently, an intramolecular vibrational redistribution (IVR) mechanism for the dissociation of $\mathrm{ArCl}_{2}$ has been proposed, in which the fragment rotational distributions are strongly dependent on the details of the vibrational structure of the complex. ${ }^{4,5}$ The sensitivity of these calculations to the assumed potential is such that one can begin to draw definitive conclusions regarding the nature (and, in particular, the anisotropy) of the van der Waals potential from a comparison between experiment and theory.

An important experimental advance in the measurement of fragment rotational distributions was achieved when Lester and co-workers demonstrated that two color pump-probe spectroscopy could be used to measure the rotational state distribution of $\mathrm{ICl}$ formed from the vibrational predissociation of $\mathrm{NeICl}$ (Refs. 6-9) and $\mathrm{HeICl}^{7,10}$ In these experiments, a "pump" laser prepares a rare gas$\mathrm{ICl}$ molecule in specified vibrational state in an excited electronic state. Vibrational predissociation occurs on a nanosecond or subnanosecond timescale, forming electronically excited $\mathrm{ICl}$ in a distribution of vibrational and rotational states. A "probe" laser, delayed in time to assure dissociation of the complex, interrogates the nascent fragment distribution by excitation to a higher lying electronic state. Total emission from the higher electronic state is detected as a function of "probe" laser wavelength, resulting in a fluorescence excitation spectrum of the ICl fragments that is sensitive to the relative populations of the internal quantum states. To date, measurements of the rotational distributions of the diatomic halogen fragment have focused on the rare gas- $\mathrm{Cl}_{2}$ (Refs. 11-17) and rare gas- $\mathrm{ICl}$ (Refs. 6-10) complexes, along with less extensive studies of the rare gas- $\mathrm{Br}_{2}$ (Ref. 18) complexes.

Motivated by these experimental investigations, numerous theoretical studies of the dynamics and bound state structure of these complexes have been carried out. Specifically, a variety of quantum mechanical calculations have explored both the nature of the quasibound states that are prepared by laser excitation and the resulting fragment rotational distributions. ${ }^{2-5,11,13,19-23}$ Both time dependent wave packet ${ }^{23}$ and time in dependent ${ }^{2-5,11,13,19-22}$ calculations have been considered. The studies have relied on van der Waals potentials constructed as sums of atom-atom pair potentials. The atom-atom potentials are assumed to have simple analytic forms (typically Morse or LennardJones), while some investigators ${ }^{3-5,11,13,20,22}$ have modified the long range form to provide the expected $1 / R^{6}$ long range radial dependence. Even with these relatively simple- 
minded potentials, the agreement between measured and calculated rotational distributions has been good.

With the goals of avoiding the computational overhead associated with full quantum mechanical calculations and of providing simple physical models for the dissociation, several approximate theoretical treatments have emerged..$^{8-10,23}$ A number of important discrepancies in the applicability of these models have arisen, however. Drobits and Lester find that the $\mathrm{ICl}$ rotational distributions that result from the dissociation of $\mathrm{NeICl}$ are consistent with those expected from a classical, inelastic "half-collision" scattering process. ${ }^{9}$ In particular, the dependence of the rotational distribution on the total energy available for disposal into the rotational and translational degrees of freedom is like that found from the dissociation of chemically bound molecules using Schinke's "rotational reflection principle."24,25 The quantum dynamics associated with the intramolecular redistribution of vibrational energy that preceeds dissociation is neglected in this model. Reasoning along these same lines, Waterland et al. investigated the classical dynamics of the $\mathrm{HeICl}$ van der Waals molecule ${ }^{10}$ and found that features in the rotational distributions can be identified as classical rotational rainbows. For this molecule, a purely impulsive classical model for the dissociation reproduces the qualitative features of the quantum state distribution. ${ }^{10}$ In addition, quantum calculations confirm a general premise of the classical models, i.e., that final states interactions dominate the disposal of rotational energy in $\mathrm{HeICl}$, while initial state preparation and vibrational coupling dynamics play a relatively minor role. ${ }^{21}$

On the other hand, Cline et al. have found that the dynamics of the $\mathrm{NeCl}_{2}$ van der Waals molecule are consistent only with quantum mechanical calculations. ${ }^{13}$ Specifically, the rotational distributions are insensitive to the total energy available for disposal into the rotational and translational degrees of freedom, suggesting that classical inelastic scattering ideas are inappropriate for this molecule. Further, a number of experimental and theoretical observations of rare gas- $\mathrm{Cl}_{2}$ complexes lead to the conclusion that neglect of the dynamics of vibrational energy redistribution can be a serious omission from any model for the dissociation of these species. ${ }^{4,5,11,13,15}$

In an earlier publication, we have described our investigation of the vibrational state distribution that results from the dissociation of the NeIBr van der Waals complex. ${ }^{26}$ We now extend this work to include higher resolution studies to extract the $\mathrm{IBr}$ fragment rotational distributions. Our goal is to lend some insight into the discrepancies noted in previous work, specifically regarding the role of available energy and "classical" concepts in modeling the dissociation of these complexes. A cursory consideration of the differences between ICl and $\mathrm{Cl}_{2-}$ containing complexes suggests several potentially important factors, including the presence of a molecular dipole moment in ICl, different ranges of available energy sampled in the two sets of data and variation in the importance of kinematic constraints on the transfer of angular momentum in the dissociation. In Sec. II, we describe the experimental apparatus and procedures used to extract IBr ro- tational distributions. In Sec. III, our experimental results are presented. Comparisons of selected rotational distributions with three models for the dissociation are described in Sec. IV, followed by a discussion of the agreement between experiment and theory in Sec. V. In our calculations, we use the model potential energy surface for $\mathrm{NeIBr}$ proposed in our earlier publication. ${ }^{26}$ Absent from this discussion is a description of the calculated quantum dynamics of this complex; these studies are in a preliminary stage and will be described at a later date.

\section{EXPERIMENT}

$\mathrm{IBr}$ rotational distributions resulting from the dissociation of the NeIBr van der Waals molecule were detected using a pump-probe excitation scheme. The wavelength of the pump laser is fixed to excite $\mathrm{NeIBr}$ to a specific vibrational level of the $A(\Omega=1)$ state using the $A \leftarrow X$ electronic transition at $\approx 720 \mathrm{~nm}$. Following a delay to assure dissociation of the complex, the probe laser interrogates the nascent $\mathrm{IBr}$ product distribution using the $G(\Omega$ $=1) \leftarrow A$ electronic transition at $\approx 308 \mathrm{~nm}$. Specifically, we measure a fluorescence excitation spectrum of the $A$ state photofragments by scanning the probe laser wavelength while detecting total emission from the $G$ electronic state. (In our previous pump-probe study of the dissociation of $\mathrm{NeIBr}$, the probe transition was the lower energy $\beta \leftarrow A$ transition at $\approx 385 \mathrm{~nm}$. While the latter scheme has the advantage that the probe laser wavelength can be generated without frequency doubling the output of our $\mathrm{N}_{2}$ pumped dye laser system, operation of the dye laser with an intercavity étalon at this wavelength proved to be impractical.) The most advantageous $G \leftarrow A$ probe transitions were identified by calculating Franck-Condon factors from the Rydberg-Klein-Rees (RKR) potential energy curves ${ }^{27}$ derived from the available spectroscopic data. ${ }^{28-30}$

The experimental apparatus used in this investigation has been described in detail in previous reports from this laboratory. ${ }^{26,31}$ Briefly, NeIBr van der Waals molecules are generated in the pulsed supersonic free jet expansion of a gas mixture composed of the room temperature vapor pressure of $\mathrm{IBr}$ and a $3 \% \mathrm{Ne}, 97 \% \mathrm{He}$ carrier gas at a total pressure of $350 \mathrm{psig}$. The expansion source is a pulsed solenoid valve (General Valve) with an orifice diameter of $150 \mu \mathrm{m}$. Our pump laser is a $\mathrm{Nd}^{3+}-\mathrm{YAG}$ pumped dye laser (Continuum Lasers YG580-30/TDL-50) operating with LD700 laser dye (Exciton) over the wavelength range $712-733 \mathrm{~nm}$ with a pulse energy of 9-13 $\mathrm{mJ}$. The probe laser is a $\mathrm{N}_{2}$-pumped dye laser (Laser Photonics UV24/ DL14P) operating with Rhodamine 610 laser dye (Exciton). With the intracavity étalon installed, this laser typically generates pulses with an energy of $\approx 300 \mu \mathrm{J}$ at 617 $\mathrm{nm}$. Tuning of the probe dye laser wavelength is accomplished by pressure scanning with $\mathrm{N}_{2}$ gas. The visible output of the probe dye laser is frequency doubled with potassium dihydrogen phosphate (KDP) in an autotracking second harmonic generator (Inrad Autotracker II) and combined with the pump laser beam using a dichroic filter. The merged pump and probe laser beams intersect the supersonic free jet expansion at a right angle to the expansion 
axis 30 nozzle diameters from the source. In a typical experiment, the delay between the pump and probe laser pulses was fixed at 100 ns. $G \rightarrow A$ laser-induced fluorescence was collected by a f/1.2 optical system and detected by an ultraviolet (UV) sensitive photomultiplier tube (Thorn/ EMI 9813QB). The photocathode was spatially masked to substantially eliminate Doppler broadening; scattered light from the pump laser was eliminated using a colored glass filter (Hoya Optics U-340). The output of the phototube was routed to a gated integrator (Stanford Research Systems SR250). Integrated fluorescence intensities were stored on a laboratory computer (Macintosh IIci) using Lab View software (National Instruments).

Previous investigations in this laboratory ${ }^{26}$ and in others $^{8,9}$ have demonstrated the ease with which the intense halogen valence-to-ion-pair electronic transitions (such as the $G \leftarrow A$ transition in IBr) can be saturated by only moderate laser pulse energies. In these investigations, after filtering out the visible dye laser fundamental, we attenuate the ultraviolet probe laser intensity with neutral density filters to assure that the probe transition is unsaturated. Typical probe laser pulse energies were $100 \mathrm{~nJ}$, though scans were recorded with pulse energies that were higher and lower to assure that the rotational distributions did not show any systematic dependence on laser pulse energy. As noted earlier, the delay between the pump and probe laser was typically fixed at $100 \mathrm{~ns}$. To assure that the observed distributions were nascent, scans were also recorded with delays as short as $10 \mathrm{~ns}$. No systematic dependence of the rotational distributions on this delay setting was observed. Finally, the photodissociation of chemically bound species is frequently accompanied by a substantial degree of alignment of the photofragments with respect to the polarization axis of the photolysis laser. ${ }^{32}$ The ramification of such an effect in the present experiments would be deviations of the relative rotational line strength factors for the probe transition from those given by the traditional HöhlLondon factors. ${ }^{33}$ To test for the presence of alignment in the dissociation of $\mathrm{NeIBr}$, we recorded rotationally resolved photofragment spectra while selectively detecting emission polarized parallel and perpendicular to the pump laser axis. (The probe and pump lasers are polarized perpendicular with respect to one another.) No systematic dependence of the spectra on the polarization of the detected emission was noted. We assume, therefore, that alignment of the $\mathrm{IBr}$ fragment is negligible.

\section{RESULTS}

In Fig. 1(a), a typical IBr photofragment excitation scan is displayed. In this scan, the pump laser is fixed on the $(12,0) A \leftarrow X$ NeIBr transition, while the probe laser wavelength is tuned over the $(0,11) G \leftarrow A$ transition in IBr. As in all such spectra, an unresolved $R$ branch bandhead is observed at high frequency, with well resolved $P$ branch features appearing at lower frequency. From Fig. 1 (a), we note that the highest rotational level detected for the $v=12 \rightarrow v=11$ dissociation channel is $j=22$. (We use the following symbols to indicate angular momenta in the dissociation of $\mathrm{NeIBr}: j$ is the diatomic fragment angular
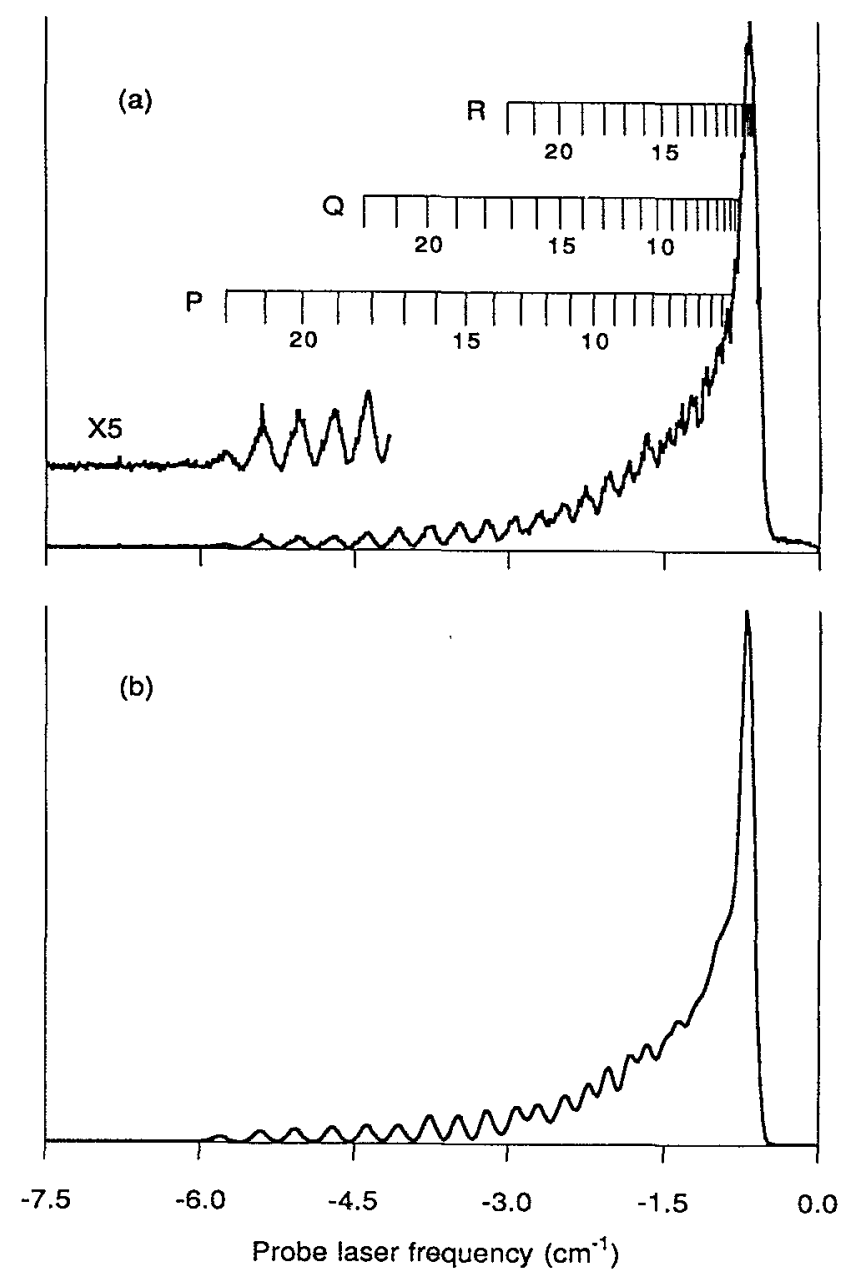

FIG. 1. $(0,11), G \leftarrow A$ fluorescence excitation scan of IBr fragments from the dissociation of $\mathrm{NeIBr}$ prepared in the $v=12$ level of the $A$ electronic state. (a) is the experimental data; (b) is a simulated spectrum using rotational state populations derived from a least-squares fit to the experimental spectrum.

momentum, $l$ is the orbital angular momentum of the recoiling fragments, and $J$ is the angular momentum of the $\mathrm{NeIBr}$ complex. In vector notation, $\mathbf{J}=\mathbf{j}+\mathbf{l}$.) To extract rotational distributions from such spectra, we compare the experimental data with simulated spectra on a point by point basis and adjust the populations of all of the observed rotational levels iteratively to achieve the best least-squares fit to the data. In Fig. 1(b) the simulated spectra that arises from our best fit to the data in Fig. 1(a) is presented.

In Fig. 2, the distribution of rotational state populations in $v=11$ that results from the dissociation of $v=12$ $\mathrm{NeIBr}$ is plotted. The filled circles represent the mean fractional populations that arise from several (typically 4-6) independent data sets. The error bars reflect the magnitudes of the scatter in the fractional populations derived from different data sets. These fluctuations are more severe for lower $j$ values $(\leqslant 10)$ since there are no cleanly resolved transitions that reveal the populations of these levels. The populations for low $j \mathrm{~s}$ are therefore more sensitive to noise fluctuations on the spectra than the populations for higher 


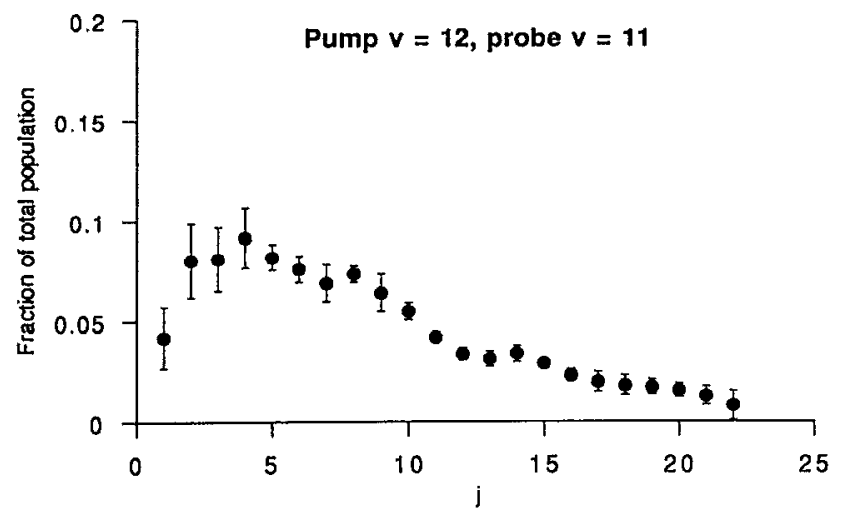

FIG. 2. Distribution of rotational states in $v=11$ following the $\Delta v=-1$ dissociation of the $v=12 \mathrm{NeIBr}$ complex. The distribution is normalized such that the sum of the populations equals 1 .

$j$ levels. We have tested our fits for sensitivity to the population distribution that we input initially in our least squares fitting procedure and find that the deviations are at most a few percent.

In Fig. 3, additional rotational distributions for $\Delta v=$ -1 dissociation processes are presented. All of the rotational distributions resulting from the loss of one vibrational quantum peak at relatively low $j(\leqslant 6)$ with gradually decreasing populations at higher $j$. In particular, the $v$ $=16 \rightarrow v=15$ distribution effectively terminates above $j$ $=10$. [Our least-squares fitting procedure assigns very
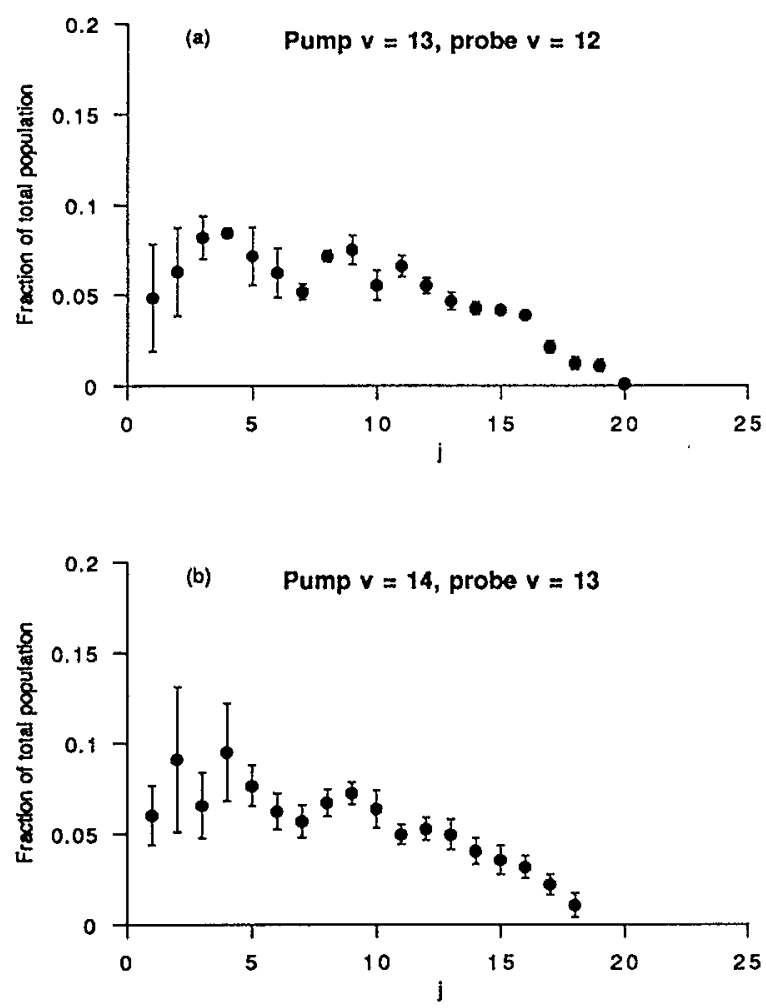

small populations to the $j=11$ and 12 levels. As indicated in Fig. 3(d), however, the error bars associated with those populations make them experimentally insignificant.] We interpret the termination of this rotational distribution as due to reaching the energetic limit for dissociation of $\mathrm{NeIBr}$ with the loss of only one quantum of I-Br vibrational excitation. In our previous study, we found that $v$ $=16$ was the highest vibrational level for which a $\Delta v=-1$ dissociation process was operative. ${ }^{26}$ The rapid change in the vibrational branching ratios (see Table II of Ref. 26) allowed us to determine the binding energy $\left(D_{0}\right)$ of the $\mathrm{NeIBr}$ complex to be $67 \pm 4 \mathrm{~cm}^{-1}$ for $v=16$ in the $A$ electronic state. Observation of a similar sharp cutoff in the rotational distribution allows us to refine our value for the binding energy. If we assume that the $\operatorname{IBr} v=15, j=10$ rotational level lies just below the dissociation threshold and that $j=11$ lies just above the threshold, then 65.1 $<D_{0} \leqslant 65.8 \mathrm{~cm}^{-1}$. This result assumes that the limiting energetic constraints are the asymptotic energies of the $\mathrm{IBr}$ fragment rotational levels. Cline et al. have noted, however, that rotational excitation of the diatomic fragment implies a degree of orbital angular momentum and a resulting centrifugal barrier in the van der Waals potential. ${ }^{13}$ For the purpose of estimating the magnitude of this effect, we follow the procedure outlined by Cline et al. and write the long range portion of the van der Waals interaction as ${ }^{13}$

$$
V(R)=-\frac{C_{6}}{R^{6}}+\frac{l(l+1) \hbar^{2}}{2 \mu R^{2}}
$$
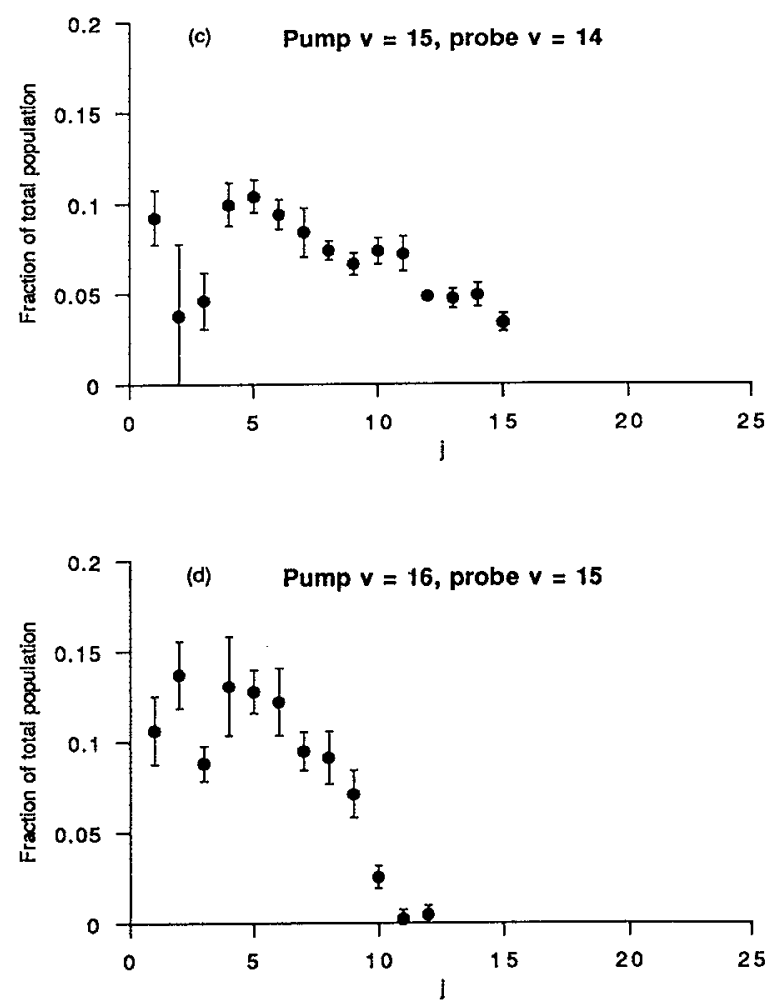

FIG. 3. Distributions of rotational states resulting from $\Delta v=-1$ dissociation events in NelBr. Each distribution is normalized such that the sum of the populations equals 1. (a) $v=13 \rightarrow v=12$; (b) $v=14 \rightarrow v=13$; (c) $v=15 \rightarrow v=14$; (d) $v=16 \rightarrow v=15$. 
TABLE I. NeIBr predissociation energetics.

\begin{tabular}{|c|c|c|c|c|c|c|c|}
\hline Initial $v$ & $\begin{array}{c}D_{0} \\
\left(\mathrm{~cm}^{-1}\right)^{\mathrm{a}}\end{array}$ & Final $v$ & $\begin{array}{c}E_{\text {avl }} \\
\left(\mathrm{cm}^{-1}\right)^{\mathrm{a}, \mathrm{b}}\end{array}$ & $\begin{array}{c}\left\langle E_{\mathrm{rot}}\right\rangle \\
\left(\mathrm{cm}^{-1}\right)\end{array}$ & $\left\langle E_{\mathrm{rot}}\right\rangle / E_{\mathrm{avl}}$ & $j_{\text {av1 }}$ & $j_{\max }$ \\
\hline \multicolumn{8}{|l|}{$\Delta v=-1$} \\
\hline 13 & 65.8 & 12 & 18.6 & 3.7 & 0.20 & 19 & 20 \\
\hline 14 & 66.1 & 13 & 13.5 & 3.2 & 0.24 & 17 & 18 \\
\hline 15 & 65.7 & 14 & 8.5 & 2.8 & 0.33 & 14 & 15 \\
\hline 16 & 65.5 & 15 & 4.1 & 1.2 & 0.29 & 10 & 10 \\
\hline 16 & 65.5 & 14 & 78.3 & 11.0 & 0.14 & 37 & 40 \\
\hline 17 & 65.3 & 15 & 68.2 & 8.9 & 0.13 & 35 & 34 \\
\hline
\end{tabular}

${ }^{a}$ Uncertainty $= \pm 1.2 \mathrm{~cm}^{-1}$.

${ }^{\mathrm{b}} E_{\mathrm{avl}} \equiv \Delta E_{\mathrm{vib}}-D_{0}$.

where $l$ is the orbital angular momentum quantum number, $R$ is the radial distance between the $\mathrm{Ne}$ atom and the IBr diatomic, and $\mu$ is the reduced mass of the triatomic system. We simplify our calculation by assuming that the initial total rotational angular momentum of the complex is zero, so that $l=j$. Note that this form also ignores any angular anisotropy of the van der Waals interaction and is thus appropriate for fragment separations that are large compared to the IBr bond length. [For example, for $l=10$ the maximum of $V(R)$ occurs at $R=10.2 \AA$.] Using this form, the height of the centrifugal barrier, $V_{\text {bar }}$, is then ${ }^{13}$

$$
V_{\text {bar }}=\left(54 C_{6}\right)^{-1 / 2}\left[\frac{l(l+1) \hbar^{2}}{\mu}\right]^{3 / 2} \text {. }
$$

We calculate $C_{6}$ assuming only dispersion interaction (the contribution from the dipole-induced dipole interaction is much smaller and is neglected for the purpose of this calculation) and using the ground electronic state electrical properties of $\mathrm{IBr}$ (Ref. 34) and Ne. ${ }^{35}$ We find that in this case $C_{6}=3.740 \times 10^{5} \mathrm{~cm}^{-1} \AA^{6}$. We then obtain,

$$
V_{\text {bar }}\left(\mathrm{cm}^{-1}\right)=5.60 \times 10^{-4}[l(l+1)]^{3 / 2} \text {. }
$$

The presence of a centrifugal barrier has the effect of making the observed dissociation energy larger than that defined using the asymptotic $\mathrm{Ne}+\mathrm{IBr}$ energies (i.e., $D_{0}$ ) by $<1 \mathrm{~cm}^{-1}$. By calculating the height of the barrier for $j=l$ $=10$ and 11 , we find that $64.2<D_{0} \leqslant 65.2 \mathrm{~cm}^{-1}$.

Further complicating our estimate of the binding energy is the likely population of excited rotational states in the $\mathrm{NeIBr}$ complex prior to dissociation. In a previous study, we have found that under the expansion conditions used, the rotational temperature of uncomplexed $\mathrm{IBr}$ is $\approx 0.9 \mathrm{~K}^{31}$ Assuming the same value for the $\mathrm{NeIBr}$ rotational temperature and a rigid rotor model for calculating the rotational constants, we estimate that van der Waals molecules in the $A$ electronic state may contain up to 1.4 $\mathrm{cm}^{-1}$ of rotational energy (i.e., $J \leqslant 5$ ). The effect of this initial rotational excitation would be to underestimate the true value of $D_{0}$ by up to $1.4 \mathrm{~cm}^{-1}$. We incorporate this possibility into our estimate by extending the range of possible dissociation energies by this amount. The final dissociation energy estimate is then $64.2<D_{0} \leqslant 66.6 \mathrm{~cm}^{-1}$ or
$D_{0}=65.5 \pm 1.2 \mathrm{~cm}^{-1}$. From the band shift of the $\mathrm{NeIBr}$ $A \leftarrow X$ excitation features relative to those from uncomplexed $\mathrm{IBr},{ }^{31,36}$ we calculate the $X$ state, $v=0$ dissociation energy to be $71.8 \pm 1.2 \mathrm{~cm}^{-1}$.

From the same band shift data, we are able to calculate the dissociation energies for all of the $A$ electronic state vibrational levels examined in this study. ${ }^{31}$ These data are presented in Table I, along with the amount of energy $\left(E_{\text {avl }}\right)$ that is available for disposal into the rotational and translational degrees of freedom. ( $E_{\mathrm{avl}} \equiv \Delta E_{\mathrm{vib}}-D_{0}$, where $\Delta E_{\text {vib }}$ is the energetic spacing between the initial and final IBr vibrational levels.) Also tabulated in Table I are the average fragment rotational energies $\left(\left\langle E_{\text {rot }}\right\rangle\right)$ and the rotational level $\left(j_{\text {avl }}\right)$ that could be populated if all of the available energy was channeled into rotation of the $\mathrm{IBr}$ fragment. $j_{\max }$ is the maximum $j$ that is observed to be populated experimentally. In our calculation of $j_{\text {avl }}$, we assume the largest value of $E_{\text {avl }}$ that is consistent with the $\pm 1.2 \mathrm{~cm}^{-1}$ error limits and use the inequality

$$
E_{\mathrm{avl}} \geqslant j_{\text {avl }}\left(j_{\text {avl }}+1\right) B_{v}+V_{\text {bar }} \text {. }
$$

(For the range of $j$ levels accessed in this study the contribution of centrifugal distortion to the rotational energy of $\mathrm{IBr}$ is negligible.) The largest value of $E_{\text {avl }}$ corresponds to the smallest value of $D_{0}$, an extreme value that does not incorporate an allowance for initial rotational excitation of NeIBr. Note from Table I that for all but the $v=16 \rightarrow 15$ dissociation channel, $j_{\mathrm{avl}}<j_{\max }$, though the difference is only one unit of rotational angular momentum. In each case, however, the discrepancy can be overcome by assuming that up to $1.4 \mathrm{~cm}^{-1}$ of NeIBr rotational energy is available for disposal into the fragments. In our analysis, we assume that the $v=16 \rightarrow v=15$ rotational distribution is limited by energetic constraints. Based on a dissociation energy derived from this one distribution, we find that the breadths of all of the rotational distributions arising from the $\Delta v=-1$ dissociation pathways extend to their energetic limits.

In Fig. 4 the photofragment rotational distributions that result when NeIBr complexes dissociate with the loss of two quanta of $\mathrm{IBr}$ vibrational excitation (e.g., $v=16 \rightarrow v$ $=14$ ) are presented. All of these distributions reveal the 

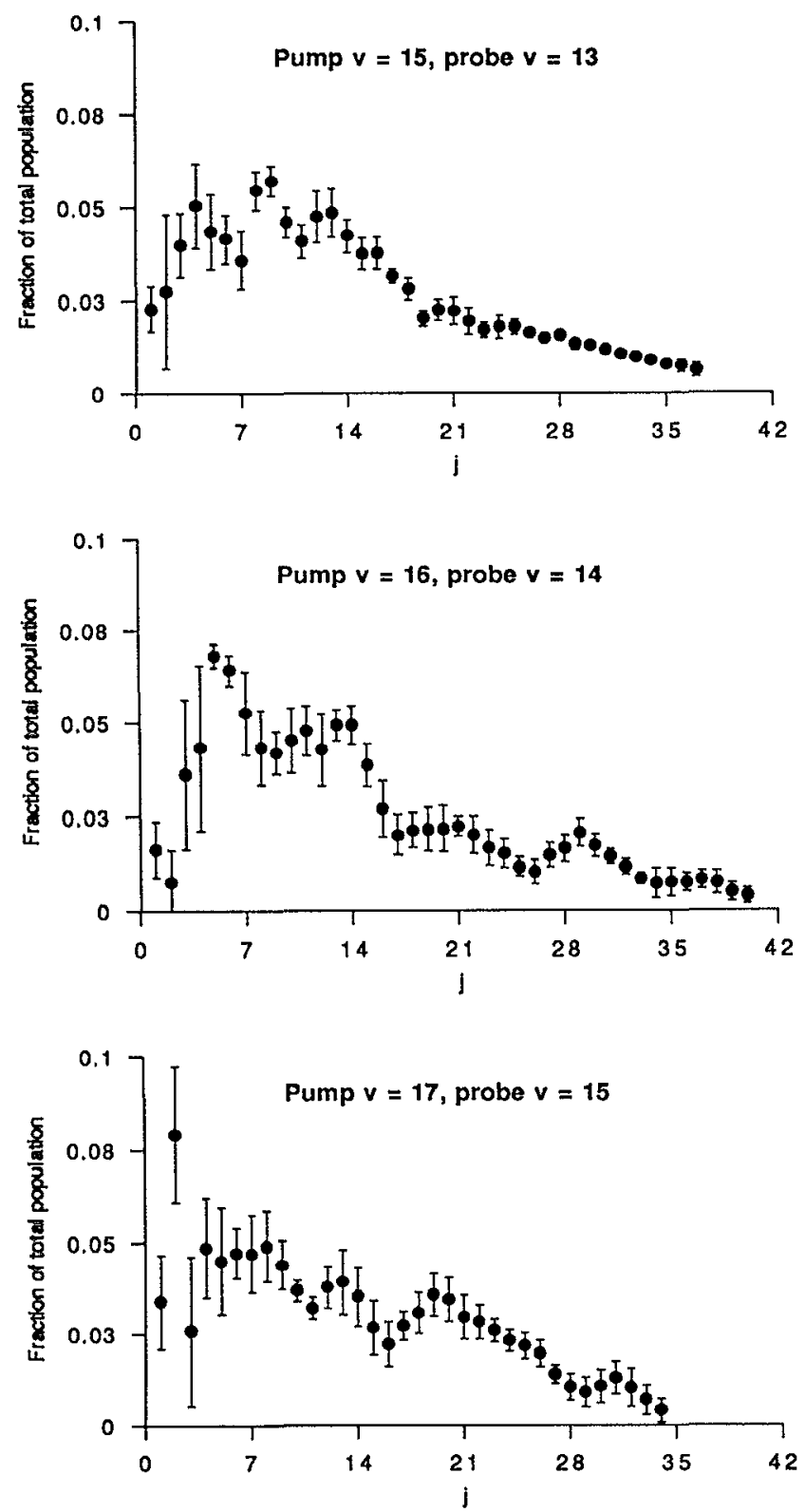

FIG. 4. Distributions of rotational states resulting from $\Delta v=-2$ dissociation events in NeIBr. Each distribution is normalized such that the sum of the populations equals 1 . (Top) $v=15 \rightarrow v=13$; (middle) $v$ $=16 \rightarrow v=14$; (bottom) $v=17 \rightarrow v=15$.

presence of fragments with significantly higher rotational excitation than any of the $\Delta v=-1$ processes. In general, however, we find (Table I) that for $\Delta v=-2$ dissociation events the fraction of the total energy available appearing in the rotational degree of freedom is on the order of half that for dissociations that occur with the loss of only one quantum of vibrational energy. Calculation of $j_{\text {avl }}$ for these dissociation events can be considered to be qualitative at best, since we calculate that the centrifugal barrier height for the highest $j$ states observed is $\sim 35 \%$ of the total available energy. Shortcomings in our simple model for the barrier are therefore magnified. In particular, for $l=35$, our model predicts that the maximum of the centrifugal barrier occurs at $R=5.6 \AA$, suggesting that our neglect of the angular dependence of the van der Waals potential is seriously compromised. A qualitative comparison of the $j_{\max }$ and $j_{\text {avl }}$ columns of Table I indicates, however, that for these dissociation channels as well the primary constraint on the breadths of the distributions appears to be energetic. The lack of a quantitative correspondence between $j_{\text {avl }}$ and $j_{\max }$ may result from the simplifications inherent in our model for the centrifugal barrier.

\section{THEORETICAL MODELS FOR IBr ROTATIONAL EXCITATION}

To explore the qualitative origin of the $\mathrm{IBr}$ fragment rotational excitation, we have considered three approximate models for the vibrational predissociation of NeIBr. While such models will in no way provide the level of detail or accuracy of, for example, quantum dynamics calculations, they do illuminate certain of the essential forces that govern the disposal of energy in the predissociation. All of our calculations are based on the model potential energy surface proposed by Walter and Stephenson for NeIBr. ${ }^{26}$ While this surface-a sum of atom-atom Morse potentials-lacks the proper $1 / R^{6}$ functional form for large radial distances, it is adequate to draw qualitative conclusions regarding the origin of rotational excitation from the approximate theoretical models under consideration. Surfaces of this form have been used with considerable success in simulations of the dynamics of $\mathrm{HeI}_{2}$ (Ref. 2), $\mathrm{HeICl}$ (Ref. 21), and NeICl. ${ }^{19}$ In addition, Gray and Wozny ${ }^{23}$ find that the addition of a long range $1 / R^{6}$ tail to additive atom-atom potentials has little impact on the lifetimes, resonance energies, and rotational distributions in quantum wave packet calculations on $\mathrm{HeCl}_{2}$ and $\mathrm{NeCl}_{2}$. Quantitative calculation of the rotational distributions from the dissociation of $\mathrm{NeIBr}$ and refinements of the potential energy surface must await the application of detailed quantum mechanical calculations.

In Sec. IV A we describe the results of quantum bound state calculations on the model $\mathrm{NeIBr}$ potential energy surface. The results of such calculations allow us to consider the validity of the Franck-Condon model for the dissociation, in which the fragment rotational state populations are derived solely from the angular dependence of the initially prepared wave function. ${ }^{37}$ In Sec. IV B, we review a semiclassical model for fragment rotational excitation in the dissociation of van der Waals molecules, as developed by Waterland et al. ${ }^{10}$ who drew on analogous developments by Schinke for chemically bound molecules. ${ }^{24,25} \mathrm{~A}$ central feature of this model is the classical excitation function $j\left(\gamma_{0}\right)$, which relates the final fragment rotational angular momentum $j$ to $\gamma_{0}$, the initial angular position of the $\mathrm{Ne}$ relative to the $\mathrm{I}-\mathrm{Br}$ axis. In Sec. IV $\mathrm{C}$ we describe the results of a classical trajectory study of the dissociation of $\mathrm{NeIBr}$ in which $j\left(\gamma_{0}\right)$ is calculated by integrating Hamilton's equations of motion. These calculations serve to illuminate the role of both the initially prepared wave function and the anisotropy of the potential energy surface on the degree of rotational excitation. The application of the classical impulsive approximation to calculate $j\left(\gamma_{0}\right)$ for the 
dissociation of NeIBr is presented in Sec. IV D. In this model, the impulsive scattering kinematics of a relatively light $\mathrm{Ne}$ atom with the massive $\mathrm{IBr}$ diatomic dominate the dynamics. In each case, our calculations focus on the $v$ $=12 \rightarrow v=11 \Delta v=-1$ dissociation (Fig. 2). We present, in addition, classical trajectory calculations on the $v=17 \rightarrow v$ $=15 \Delta v=-2$ dissociation.

\section{A. Bound state calculation and the Franck-Condon model}

As noted in our introductory remarks, quantum bound state and dynamics calculations have proven to be a valuable tool in our understanding of the behavior of rare gas/ halogen van der Waals complexes. ${ }^{2-5,11,13,19-23}$ We have applied the programs of Halberstadt and co-workers ${ }^{3,19-22}$ to the NeIBr system using our model potential energy surface. The theoretical methodology on which these calculations are based was developed by Halberstadt, Beswick, and co-workers and has been described elsewhere in considerable detail. ${ }^{3,19-22}$ Briefiy, in this scheme the Hamiltonian operator is written in terms of the internal coordinates $r$, the magnitude of the vector from $\mathrm{I}$ to $\mathrm{Br}, R$, the magnitude of the position vector of the $\mathrm{Ne}$ atom relative to the $\mathrm{IBr}$ center-of-mass and $\gamma$, the angle between these two vectors. The higher frequency $\mathrm{IBr}$ vibrational motion is separated from the lower frequency van der Waals vibrational motion by calculating the bound state energies and wave functions for an effective $\mathrm{Ne}-\mathrm{IBr}$ potential in which our model potential $V(r, R, \gamma)$ is averaged over the $\mathrm{IBr}$ vibrational motion. Using this approximation, the averaged Hamiltonian $H_{v, v}$ for the NeIBr complex that correlates with IBr vibrational level $v$ is written as, ${ }^{3,19-22}$

$$
H_{v, v}=-\frac{\hbar^{2}}{2 \mu} \frac{\partial^{2}}{\partial R^{2}}+\frac{l^{2}}{2 \mu R^{2}}+V_{v, v}(R, \gamma)+E_{\mathrm{IBr}}(v)+\frac{B_{v}}{\hbar^{2}} j^{2} .
$$

$V_{v, v}(R, \gamma)$ is the averaged intermolecular van der Waals potential, while $E_{\mathrm{IBr}}(v)$ is the vibrational eigenvalue for uncomplexed IBr. $B_{v}$ is the relevant IBr rotational constant and $\mu$ is the reduced mass of the Ne-IBr system. For these calculations we fix the total angular momentum $J$ to $0 .^{37}$

The desired wave functions, $\psi_{k, v}(R, \gamma)$ which describe the motion of the $\mathrm{Ne}$ atom relative to $\mathrm{IBr}$, are the eigenfunctions of $H_{v, v^{*}}$ This Hamiltonian is diagonalized using a finite two dimensional $(R, \gamma)$ basis set, with each basis set function written as a product of an angular and a radial function. ${ }^{3,19-22}$ The radial functions are harmonic oscillator functions, while the angular functions are obtained by prediagonalization of the angular part of $H_{v, v}$ using a basis set of $\mathrm{IBr}$ free rotor functions at a specified $R=R_{e}=4.1 \AA$. For $J=0$, these functions are simply appropriately normalized spherical harmonics, $Y_{j, 0}(\gamma)$. The detailed composition of the basis functions is described in Refs. 19-22. In our calculations, we use 15 harmonic oscillator functions to describe the $R$-dependence of the $\mathrm{Ne}$ wave function along with 15 bending basis functions, each composed of $30 \mathrm{IBr}$ free rotor functions. The bound state calculations described here were carried out on a Silicon Graphics 4D/ 440VGX workstation at the University of Pennsylvania and required $\sim 11 \mathrm{~min}$ of CPU time.
As a preliminary test of the model van der Waals potential energy surface, we consider the predicted dissociation energy $D_{0}$. The calculated value is found to be 66.0 $\mathrm{cm}^{-1}$ for the $A$ state, $v=12$, a value that is in exact agreement with the experimentally determined figure of 66.3 $\pm 1.2 \mathrm{~cm}^{-1}$. Thus, prior to considering any of the dynamics supported by this potential surface we have strong evidence that the shape and depth of the potential minimum is at least qualitatively correct.

In the Franck-Condon model for the dissociation of chemically bound molecules, rotational excitation of the fragments arises from the zero-point bending motion that is initially prepared prior to dissociation. ${ }^{24,38}$ This model has proved to be particularly effective at reproducing the rotational distributions that arise from the direct dissociation of molecules in which the energy available for fragment excitation is large compared to features on the molecular potential energy surface. ${ }^{24}$ Application of the Franck-Condon model to the dissociation of NeIBr is particularly facile because we have chosen to represent the wave function of the quasibound $\mathrm{Ne}$ atom in terms of the $\mathrm{IBr}$ free rotor basis set. In this case, the probability of a particular fragment rotational state is simply the squared amplitude of the expansion coefficient in the expression for the initially excited angular wave function. In Fig. 5(a), we present the result of applying such a model to the dissociation of $\mathrm{NeIBr}$ from the $A$ electronic state, $v=12$.

\section{B. Semiclassical model for vibrational predissociation}

A semiclassical model for the dissociation of the $\mathrm{HeICl}$ van der Waals molecule has been developed by Waterland et al. ${ }^{10}$ In this approach, the van der Waals complex is assumed transferred from the initially prepared quasibound level correlating with the $\mathrm{IBr}$ vibrational level $v$ to the repulsive wall of the van der Waals potential correlating with vibrational level $v-1$. No attempt is made to account for the intramolecular redistribution of vibrational energy that must accompany this event. The probability of such redistribution is assumed to be independent of the radial $(R)$ and angular $(\gamma)$ position of the Ne atom relative to IBr. The angle $\gamma$ is conserved upon transfer to the repulsive surface; we label this initial angle $\gamma_{0}$. (See Sec. IV A for the definition of these coordinates.) The entire fragment rotational distribution then arises from the scattering, at energy $E_{\text {avl }}$, from the repulsive wall of the $v-1$ van der Waals potential. In the paragraphs that follow, we review two models that have been used to represent the scattering process. ${ }^{10}$ Common to both is the calculation of the classical excitation function $j\left(\gamma_{0}\right)$, the form of which is an indication of the angular anisotropy of the potential surface. If, for example, the van der Waals potential were completely isotropic in the angular dimension, the final diatomic angular momentum $(j)$ would be independent of initial angle $\gamma_{0}$. On the other hand, a highly anisotropic potential results in a strong dependence of the final angular momentum on $\gamma_{0}$.

Waterland et al. ${ }^{10}$ implement the semiclassical model for vibrational predissociation by drawing on analogous 

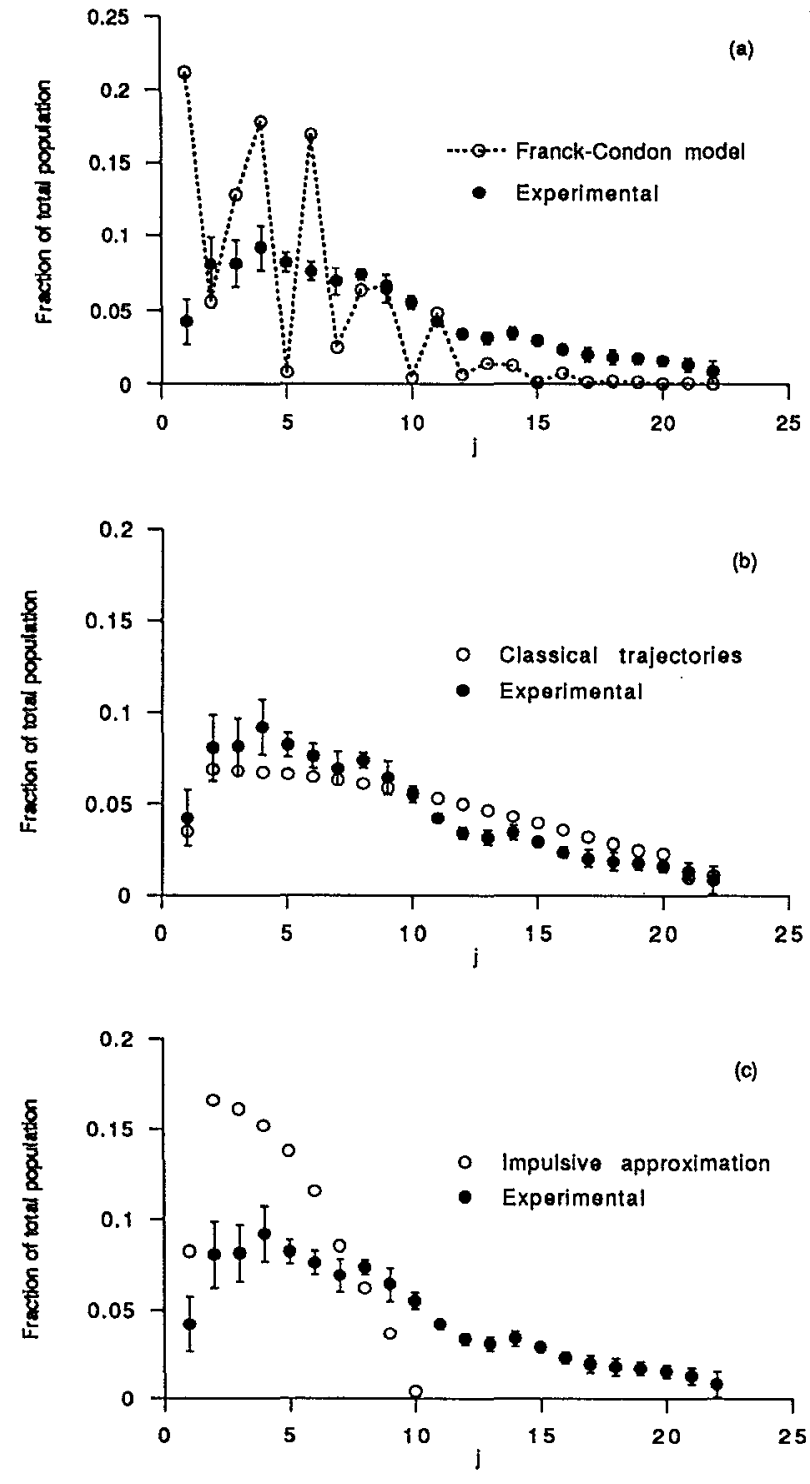

FIG. 5. Theoretical models for $\mathrm{IBr}$ fragment rotational excitation (open circles) compared with the experimental data (solid circles) for the $\Delta v$ $=-1$ dissociation of $v=12 \mathrm{NeIBr}$. (a) Franck-Condon model; (b) classical trajectory calculations; (c) classical impulsive approximation.

studies by Schinke on the photodissociation of chemically bound molecules. ${ }^{24,25}$ Assuming that the initial linear and rotational angular momentum are zero, Schinke finds that the classical cross section can be written as

$$
\sigma(j)=\sum_{\alpha} \sin \gamma_{0, \alpha} W\left(\gamma_{0, \alpha}\right)\left|\frac{d j}{d \gamma_{0}}\right|_{\gamma_{0, \alpha}}^{-1}
$$

in which the summation over $\alpha$ includes all of the angles $\gamma_{0}$ that give rise to a particular integral value of the angular momentum $j . W\left(\gamma_{0}\right)$ is a weighting factor that accounts for the fact that not all initial angles $\gamma_{0}$ are equally probable. In the present calculations, $W\left(\gamma_{0}\right)=\left|\psi_{k, v}\left(R_{e}, \gamma\right)\right|^{2}$, i.e., we use the bound state probability distribution determined in our quantum mechanical calculation (evaluated at the equilibrium van der Waals bond length, $R_{e}$ ) as the initial angular distribution. The inverse derivative term in $\mathrm{Eq}$.
(1) generates classical singularities in the distribution of rotational states for those cases near the extrema of the excitation function. These rotational rainbow structures have been shown to occur in both the photodissociation dynamics of chemically bound molecules ${ }^{39}$ and the van der Waals complex $\mathrm{HeICl}{ }^{10}$

\section{Classical trajectory calculations}

In this section, we describe our calculation of the classical excitation function by propagating exact classical trajectories on the model potential energy surface. In these calculations and in our analysis, we follow closely the previous work of Schinke ${ }^{24,25}$ and Waterland et al. ${ }^{10}$ Briefly, we choose the initial fragment linear and angular momentum to be zero. The energy of the trajectory is set to $E_{\mathrm{ayl}}$. For a particular choice of initial angle $\gamma_{0}$, the corresponding value of the radial distance $R_{0}$ is found from the condition

$$
V_{\mathrm{vdw}}\left(R_{0}, \gamma_{0}\right)=E_{\mathrm{avl}}
$$

such that the Ne atom is motionless on the repulsive wall of the van der Waals potential at the initiation of each trajectory. The total angular momentum, $J$, is set to 0 so that any fragment diatomic rotational angular momentum is exactly counterbalanced by orbital motion of the recoiling fragments. $\gamma_{0}$ values are chosen to lie on a uniform grid between 0 and $\pi$. We integrate Hamilton's equations of motion for a total of $50 \mathrm{ps}$ for each trajectory, which is found to be sufficient for $j(t)$ to reach its asymptotic value. For these calculations, we evaluate the potential energy by holding the $\mathrm{I}-\mathrm{Br}$ bond length fixed at the $A$ state $v=11$ average value of $3.029 \AA$.

The resulting classical excitation function is shown in Fig. 6(a), in which we have included only the most relevant angular range. [In this figure we have plotted $|j|$ (in units of $\hbar$ ), since our experiments are insensitive to the absolute sign of the angular momentum. The two maxima which appear on this plot occur at $j$ values of opposite absolute sign, such that one is a minimum and one is a maximum. The angular momentum $j$ is a continuous function but changes sign at $\gamma_{0}=73^{\circ}$ such that the apparent minimum in Fig. 6(a) is an artifact of choosing to plot $|j|$.] Also plotted in Fig. 6(a) is the $\mathrm{Ne}$ atom angular probability distribution, as determined from the bound state calculation. The latter plot demonstrates that the $\mathrm{Ne}$ atom is approximately confined to the angular region between $60^{\circ}$ and $85^{\circ}$. $\left(0^{\circ}\right.$ corresponds to the linear $\mathrm{I}-\mathrm{Br}-\mathrm{Ne}$ configuration.) The angles corresponding to extrema in the classical excitation function are seen to be on the periphery of this range, so that we do not expect significant rotational rainbow features for this dissociation. To calculate the expected rotational distributions, we use Eq. (1) by fitting the excitation function with a cubic spline function to evaluate the relevant derivatives. The resulting rotational distribution is plotted in Fig. 5(b), along with the experimental data for the $v=12 \rightarrow v=11$ dissociation. In Fig. 7, we present analogous classical trajectory calculations for the $v=17 \rightarrow v=15$ dissociation. 

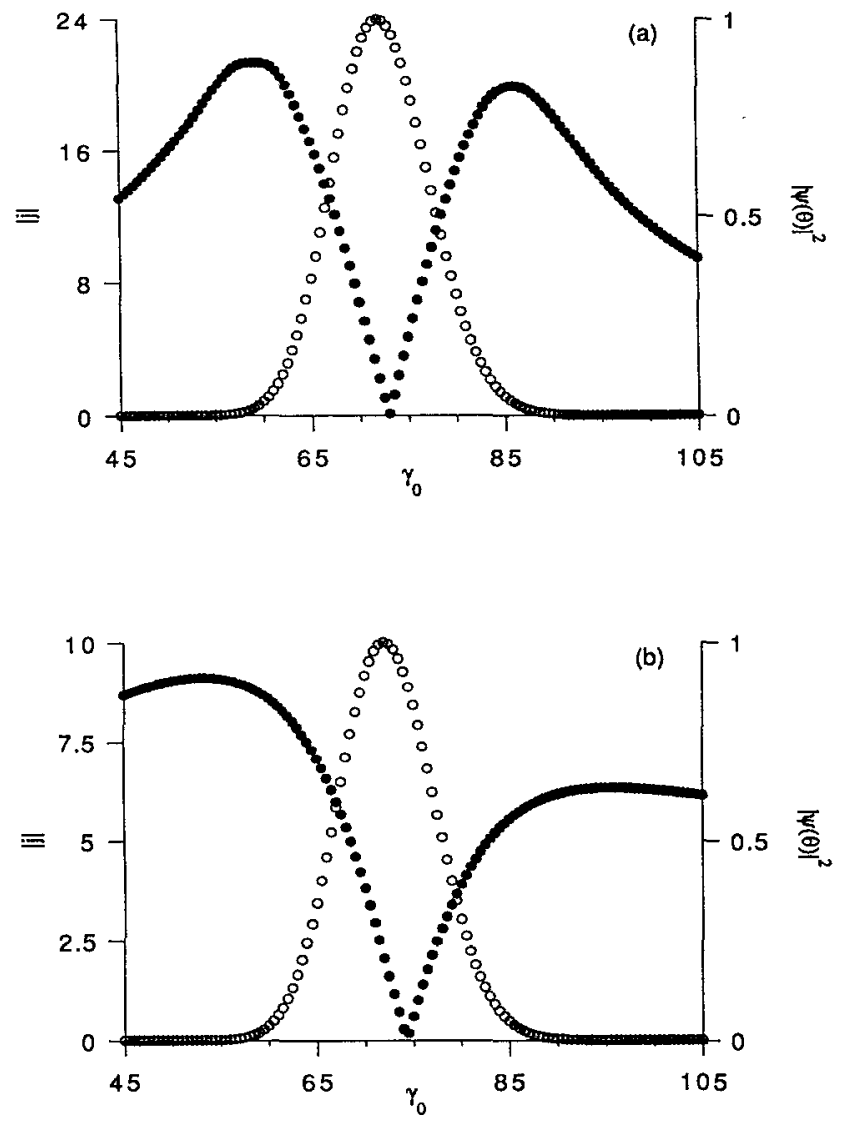

FIG. 6. Classical excitation functions (solid circles) and Ne atom angular probability distributions (open circles) used in two classical models for the dissociation of NeIBr. The initial $\mathrm{Ne}-\mathrm{IBr}$ angle $\gamma_{0}$ is plotted in degrees. The magnitude of the angular momentum, $|j|$, is plotted in units of $\hbar$. The probability distributions are normalized such that the maximum value is 1.0 . (a) Classical trajectory calculations; (b) classical impulsive approximation.

\section{Classical impulsive approximation}

As a test of the relative importance of simple classical kinematics and final state interactions in determining the fragment quantum state distribution, we have also calcu-

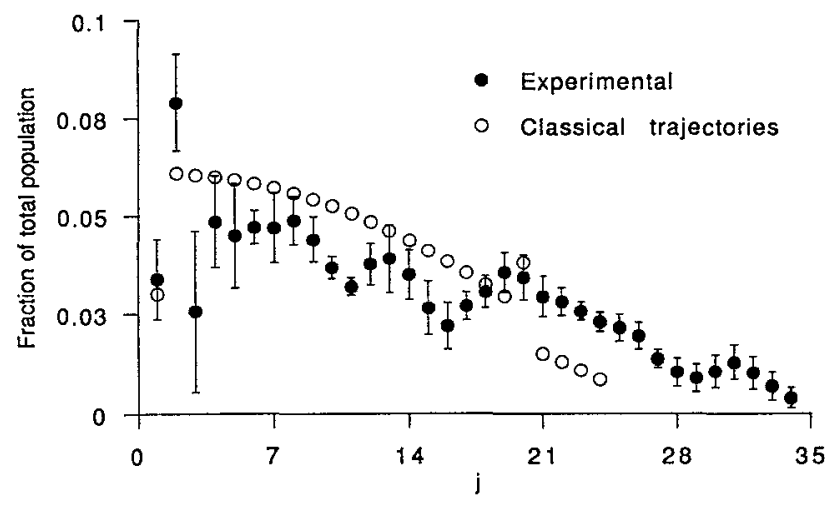

FIG. 7. Classical trajectory calculation of IBr fragment rotational excitation (open circles) compared with the experimental data (solid circles) for the $\Delta v=-2$ dissociation of $v=17 \mathrm{NeIBr}$. lated the excitation function $j\left(\gamma_{0}\right)$ as if the interaction were entirely impulsive in nature. In this calculation we simplify the potential by including only the angular form of the contour that defines the repulsive wall at an energy of $E_{\text {avll }}$. The Ne atom scatters from this contour as if the only source of rotational excitation were the sudden impulsive "kick" imparted by the recoiling fragments. In this situation, the $\mathrm{IBr}$ attains its final rotational angular momentum instantaneously and we determine this value from the expression $^{10}$

$$
j^{2}=\frac{E_{\text {avl }}}{B_{v}+1 / 2 \mu b^{2}}
$$

by calculating the effective impact parameter $(b)$ associated with each initial atomic configuration.

In Fig. 6(b), the classical excitation function that we obtain from this model for rotational excitation is presented. Note in particular that the degree of rotational excitation is much diminished relative to that arising from the trajectory calculations. In addition, the weaker angular dependence of the repulsive wall as compared to the full potential leads to an excitation function that is a less sharply peaked function of $\gamma_{0}$. The rotational distribution predicted using this model is calculated using Eq. (1) and shown in Fig. 5(c) along with the experimental data.

\section{DISCUSSION}

In this section we discuss the conclusions that we draw based on the theoretical models and their degree of agreement with the experimental $\mathrm{IBr}$ rotational distributions. While the numerous assumptions inherent in each of models dictate that only qualitative aspects of the problem are addressed, we believe that a number of useful features emerge from the comparison. Throughout this discussion, we draw on additional comparisons of the experimental data with similar results from the dissociation of the $\mathrm{NeCl}_{2}, \mathrm{NeICl}$, and $\mathrm{NeBr}_{2}$ van der Waals molecules. We conclude this section with some comments on the applicability of the "energy gap" law.

\section{A. Franck-Condon model}

Figure 5(a) documents the failure of the FranckCondon model to reproduce the IBr fragment rotational distribution. The calculated distribution is significantly narrower than that observed experimentally. Not surprisingly, the approximations inherent in the Franck-Condon model are inappropriate for the case of $\mathrm{NeIBr} \rightarrow \mathrm{Ne}+\mathrm{IBr}$. In particular, the low value of the available energy (22.9 $\mathrm{cm}^{-1}$ ) as compared to the magnitude of the van der Waals interaction potential $\left(D_{0}=66.3 \mathrm{~cm}^{-1}\right)$ render the neglect of final states interactions highly suspect. We conclude from these results that while the contribution of the zeropoint bending motion to the final fragment rotational excitation is not negligible, it is insufficient to account for the breadth of the observed distribution.

The oscillations apparent in the distribution predicted by the Franck-Condon approximation arise from the nearsymmetry of the wave function about the equilibrium bond 
angle. As noted previously by Roncero et al., free rotor wave functions that contain a node in that region have small or negligible contributions to the initial bending wave function. ${ }^{19}$ While these oscillations are clearly not reproduced in the experimental results, we attribute this discrepancy to our restriction to $J=0$ in the calculations. We anticipate that contributions from $J \neq 0$ will tend to obscure such features so that a properly weighted combination of contributions from the populated $J$ states will be significantly smoother.

Cline et al. found the Franck-Condon model useful to account for certain features of the distribution of $\mathrm{Cl}_{2}$ rotational states populated in the dissociation of $\mathrm{NeCl}_{2} \cdot{ }^{13} \mathrm{In}$ particular the relative invariance of the rotational distributions to the initial vibrational level and the number of $\mathrm{Cl}_{2}$ vibrational quanta lost is consistent with a static constraint (such as the form of the quasibound angular wave function) on the dissociation. The prevalence of low rotational levels was also found to be consistent with a decomposition of the wave function into fragment free rotor functions. The model did not reproduce either the breadth or bimodal structure of the $\mathrm{Cl}_{2}$ distributions, however. ${ }^{13}$ For NeIBr, the degree of agreement between experiment and the Franck-Condon model is similar to that for $\mathrm{NeCl}_{2}$. Ignoring the oscillatory structure in the predicted distribution, the Franck-Condon model predicts a distribution peaking at low $j$, with a steady decrease in population for higher angular momentum states. While the overall breadth is poorly represented, the $\mathrm{IBr}$ distributions could be viewed as qualitatively similar to one another (for low rotational states), but with different energetic limits. In contrast to the case of $\mathrm{NeCl}_{2}$, the small vibrational spacings in $\mathrm{IBr}$ dictate that we are unable to examine rotational distributions that are unambiguously free of energetic limitations on the extent of rotational excitation.

The Franck-Condon model has also been applied to the dissociation of $\mathrm{NeICl}$, with calculated distributions that are substantially in accord with those described here. ${ }^{19}$ A significant difference lies in the comparison with experiment, however. For some $\mathrm{NeICl}$ dissociation events, the rotational distribution peaks at intermediate values of $j{ }^{7}$ such that there is no correspondence between the predicted and experimentally observed distributions. For example, from Figs. 3 and 5 of Ref. 19, we note that the model predicts significant population in $j \leqslant 10$ only, while the experimental distribution is inverted and peaks at $j=15-18$.

The Franck-Condon model predicts qualitatively similar degrees of rotational excitation from the dissociation of the molecules $\mathrm{NeCl}_{2}, \mathrm{NeICl}$, and $\mathrm{NeIBr}$, a result of the similarity of the model potential energy surfaces near the minimum energy configuration. (All three surfaces are represented as the sum of two Morse atom-atom pair potentials.) The breadth of the Franck-Condon predicted rotational distribution is inversely related to the angular range of the zero-point bending wave function. For example, an interaction potential that is isotropic in the angular coordinate arises when the diatom can execute free rotation within the complex and would generate a "bending" wave function consisting of exactly one free rotor state function. The breadth of the calculated distribution is, however, insensitive to the angular position of the minimum relative to the diatomic internuclear axis, a feature that will be critical in determining the degree of impulsive excitation of the diatom by the recoiling $\mathrm{Ne}$ atom. When applied to a series of van der Waals molecules, the FranckCondon model is thus unable to take into account potentially important effects such as mass and/or geometric asymmetries that can affect the distribution of fragment rotational states. It is, therefore, consistent with the limitations of the model that $\mathrm{NeICl}$ photofragments exhibit the largest deviations. Because asymmetry effects will be smaller for $\mathrm{NeIBr}$, we might expect that the contribution of the bending state wave function to the experimental rotational distribution will be qualitatively similar to that found for $\mathrm{NeCl}_{2}$, in accord with the trends we have identified.

\section{B. Semiclassical models}

Figures 5(b) and 5(c) illustrate that a simple impulsive model for the dissociation fails to predict the breadth of the fragment rotational distribution, while the exact classical trajectory calculation provides an excellent fit to the data. This discrepancy points to the absolute importance of considering the dynamics supported by the entire potential energy surface in problems involving low total energy and/or significant potentials wells. Recall that classically, the change in the angular momentum with time is directly related to the angular anisotropy of the potential. The rotational excitation of the IBr fragment arises from consideration of the full anisotropy of the potential (not just that of the repulsive wall), combined with an appropriate weighting of the initial angular configurations. A cursory consideration of the dissociation of a light atomheavy diatom triatomic molecule (such as $\mathrm{Ne}-\mathrm{IBr}$ ) might suggest that the amount of fragment rotational excitation will be severely limited. These kinematic considerations dominate the narrow rotational distribution that arises from the classical impulsive model for the dissociation. The divergence of the full classical trajectory and impulsive results serves as warning that such elementary concepts should be used with considerable caution.

The adequacy of the classical trajectory calculation is reminiscent of the degree of agreement found by Waterland et al. for the case of $\mathrm{HeICl} .^{10}$ Of the rare gas/halogen van der Waals complexes examined to date with rotational resolution of fragment quantum states, we expect that $\mathrm{NeIBr}$ will be the most "classical" system, as the assumption of continuous angular momentum values will be least severe when the rotational constant of the diatom is small. Calculations based on this one $\mathrm{NeIBr}$ rotational distribution allow us to conclude that the semiclassical model developed by Waterland et al. ${ }^{10}$ may have general utility for complexes containing the heaviest halogen diatomics.

An additional symptom of classical behavior is suggested by the work of Drobits and Lester on $\mathrm{NeICl}$, in which the $\mathrm{ICl}$ rotational distributions are found to scale with $E_{\mathrm{avl}}$ in a manner that is consistent with classical halfcollision scattering. ${ }^{9}$ We are unable to apply similar con- 
siderations to NeIBr. All of the rotational distributions obtained are limited by energetic constraints, so that scaling relationships are difficult to deduce. An alternative test of the classical model would be to determine whether the fit between experiment and classical trajectories is independent of the available energy. This test is difficult to carry out for NeIBr because of the quite small values of $E_{\text {avl }}$ that are accessible. For $\Delta v=-1$ transitions that have lower available energy than that considered in Fig. 5 (e.g., $v$ $=13 \rightarrow v=12 ; E_{\mathrm{avl}}=18.6 \mathrm{~cm}^{-1}$ ), the classical excitation function is no longer smooth. The trajectories show evidence of orbiting resonances, in which the $\mathrm{Ne}$ atom obtains a high degree of orbital angular momentum early in the trajectory and is unable to escape the centrifugal barrier. Eventually, dissociation occurs following a second encounter between the fragments in which their respective angular momenta are quenched. The result is a final angular momentum that is no longer a smooth function of initial angle $\gamma_{0}$. The actual form of the classical excitation function is similar to the fractal pattern identified by Noid et al. in their classical studies of vibrational energy transfer in inelastic collisions of He with $\mathrm{I}_{2}{ }^{40}$ The simplified model described by Eq. (1) is, therefore, not applicable for $\Delta v=-1$ dissociation events other than the one indicated in Fig. 5.

We have applied the classical trajectory method to the $\Delta v=-2$ dissociation in which $v=17 \mathrm{NeIBr}$ yields $v=15$ IBr fragments (Fig. 7). Two features of the calculated distribution are of interest. First, the local maximum in the calculated rotational level populations at $j=20$ corresponds to a classical rotational rainbow in which the enhancement of the population has been largely damped. This result arises from the sharply decreasing amplitude of the bound state wave function for initial angles $\gamma_{0}$ that yield maximum fragment rotational excitation. (The maxima in the classical excitation function for this dissociation occur at $|j|=20$ and 24.) Second, the calculated distribution is narrower than that experimentally observed. Specifically, the average fragment rotational energy predicted by the classical trajectory method $\left(4.8 \mathrm{~cm}^{-1}\right)$ is significantly smaller than observed $\left(8.9 \mathrm{~cm}^{-1}\right)$. Assuming that the classical approach is a reasonable model for final state interactions, this result may arise from one (or more) of several approximations in our calculations. First, the model potential energy surface may be insufficiently anisotropic to generate the high fragment angular momentum states observed experimentally. A more anisotropic potential might be expected to improve agreement for the $\Delta v=-2$ channel while maintaining the level of agreement with the $v=12 \rightarrow v$ $=11 \Delta v=-1$ distribution, since the breadth of the latter calculated distribution is limited by an energetic constraint. Second, the calculations are restricted to $J=0$, while we estimate that angular momentum states as high as $J=5$ are populated in the free jet expansion. Lifting this restriction would undoubtedly broaden the distribution. We note, however, that the breadth of the $v=12 \rightarrow 11$ distribution was reproduced by considering only $J=0$ contributions; it is not clear why this assumption might dramatically fail in one case but not another. Third, when applied to a $\Delta v=-2$ dissociation pathway, the classical model im- plicitly assumes a direct transfer between the initial $v$ and final $v-2$ potential energy surfaces. The dynamics are then solely determined by propagation on the $v-2$ surface of a distribution of initial conditions derived from the bound state wave function correlating with vibrational level $v$. Halberstadt et al. have identified IVR as an important feature in the dissociation of the $\mathrm{ArCl}_{2}$ van der Waals molecule. ${ }^{4,5}$ In this mechanism, the initial state $v$ interacts with the bound states of the $v-1$ surface, followed by subsequent dissociation of the mixed state on the $v-2$ surface. While the lack of erratic structure in the rotational distributions (as observed for $\mathrm{ArCl}_{2}$ ) (Ref. 15) suggests that IVR may not be important, we are unable to either confirm or eliminate the IVR mechanism for NeIBr. Clearly the classical model fails to incorporate this potentially important feature. For other $\Delta v=-2$ events (i.e., $v=15 \rightarrow v=13$ and $v=16 \rightarrow v=14$ ), IVR is not possible because the $\Delta v=$ -1 channels are also energetically open, though with small values of $E_{\text {avy }}$. Our classical trajectory calculations indicate that at these energies there are long-lived orbiting resonances on the $v-1$ surface. The quantum mechanical manifestations of these structures has not been explored; the classical model simply neglects any intermediate role for the $v-1$ surface.

\section{Energy gap law analysis}

The "energy gap" or "momentum gap" relations have proved to be important qualitative guides in predicting the disposal of internal energy within the fragments from the dissociation of van der Waals molecules. ${ }^{41,42}$ Ewing has demonstrated that under certain conditions they have quantitative value when comparing the predissociation lifetimes of a series of systems with varying degrees of vibrational energy transfer. ${ }^{42} \mathrm{~A}$ quantitative means of predicting the degree of fragment rotational excitation has also been developed and applied to the $\mathrm{NeBr}_{2}$ and $\mathrm{HeBr}_{2}$ complexes. ${ }^{18}$ Agreement between the experimentally measured rotational distribution and that predicted by the energy gap law is poor, with the model predicting significantly less excitation than observed. When the overall propensity for fragment rotational excitation is calculated, however, for the series $\mathrm{HeCl}_{2}, \mathrm{NeCl}_{2}, \mathrm{HeBr}_{2}$, and $\mathrm{NeBr}_{2}$, the trends are in accord with the experimental observations. ${ }^{18}$

Briefly, Ewing relates the predissociation lifetime to the degree of overlap between the initial quasibound and final fragment wave functions. ${ }^{42}$ Since the overlap will be poor for wave functions with very different numbers of nodes, those dissociation channels that result in small overall changes in quantum numbers will be favored. For example, if the presence of fragment rotation is neglected, $\Delta v=-1$ channels are favored over $\Delta v=-2$ channels (assuming both are energetically open). This result arises from both the smaller change in the vibrational quantum number and, more importantly, the much smaller change in the amount of energy released to translation.

In an earlier publication, we noted that reasoning along these lines suggests that the predissociation lifetime of $\mathrm{NeIBr}$ should increase at the vibrational level that the $\Delta v=-1$ channel became energetically inaccessible. ${ }^{26}$ Such 


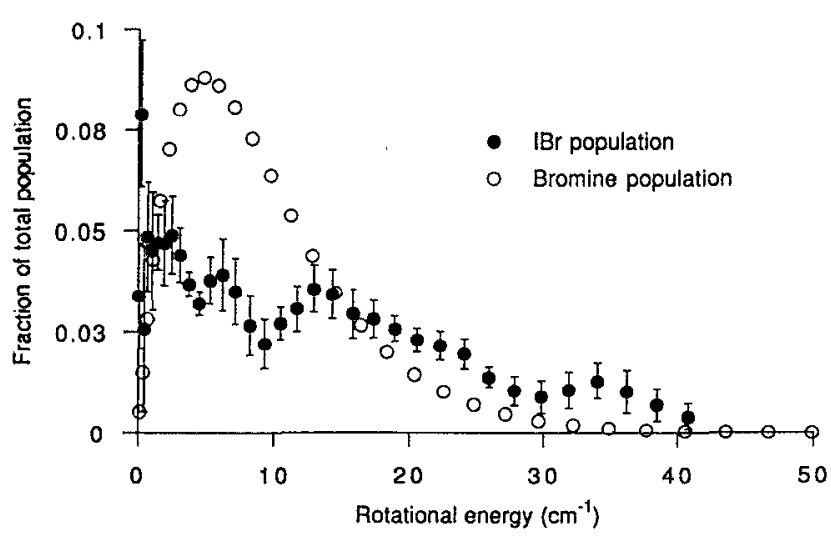

FIG. 8. Distributions of diatomic fragment rotational states from the dissociation of $\mathrm{NeIBr}$ (solid circles, $E_{\mathrm{ayl}}=68.2 \mathrm{~cm}^{-1}$, this work) and $\mathrm{NeBr}_{2}$ (open circles, $E_{\mathrm{avl}}=70 \mathrm{~cm}^{-1}$, Ref. 18).

an effect has been observed in the vibrational state dependence of the predissociation lifetimes of $\mathrm{NeBr}_{2}$ (Ref. 43) but is absent in NeIBr. As noted earlier, this analysis is dependent on a neglect of the fragment rotational degree of freedom; we suggested that for $\mathrm{NeIBr}$ this approximation may not be valid and that the excitation of fragment rotation is greater for $\mathrm{NeIBr}$ than for $\mathrm{NeBr}_{2}$. Qualitatively, the observation of rotational distributions that effectively extend to the energetic limit is confirmation of this suggestion.

On a quantitative level, Sivakumar et al. measured the rotational distribution resulting from the dissociation of $\mathrm{NeBr}_{2}$ in the $B$ electronic state and found that the populations are well characterized by a Boltzmann distribution with a temperature of $6.9 \mathrm{~K} .{ }^{18}$ The dissociation channel examined, $v=10 \rightarrow v=9$, has an available energy of 70 $\mathrm{cm}^{-1}$, close to that $\left(68.2 \mathrm{~cm}^{-1}\right)$ of the NeIBr $v=17 \rightarrow v$ $=15$ dissociation pathway. These rotational distributions are compared in Fig. 8. In this plot, the horizontal axis is rotational energy, not rotational quantum number, so that the effect of differing rotor constants is eliminated. The $\mathrm{Br}_{2}$ distribution is significantly narrower than that for $\mathrm{IBr}$, a result that can also be observed by comparing the average $\mathrm{Br}_{2}$ fragment rotational energy $\left(\left\langle E_{\mathrm{rot}}\right\rangle=5 \mathrm{~cm}^{-1} ;\left\langle E_{\mathrm{rot}}\right\rangle\right\rangle$ $E_{\text {avl }}=0.07$ ) (Ref. 18) with that for $\operatorname{IBr}\left(\left\langle E_{\mathrm{rot}}\right\rangle=8.9 \mathrm{~cm}^{-1}\right.$; $\left.\left\langle E_{\mathrm{rot}}\right\rangle / E_{\mathrm{avl}}=0.13\right)$.
The comparison of $\mathrm{NeIBr}$ and $\mathrm{NeBr}_{2}$ photofragment distributions is valid only if the mechanism of the $\Delta v=-2$ dissociation of $\mathrm{NeIBr}$ is qualitatively similar to the $\Delta v=$ -1 dissociation of $\mathrm{NeBr}_{2}$. As noted earlier, Halberstadt et al. have identified IVR in the dissociation of the $\mathrm{ArCl}_{2}$ complex. ${ }^{4,5}$ If NeIBr dissociates with the loss of two quanta of vibrational excitation by a nondirect (i.e., sequential) mechanism, a number of factors-such as the nature of the intermediate state-complicate drawing conclusions from either trends in the predissociation lifetimes or comparisons of rotational distributions. In the preceding discussion and in what follows, we assume a direct dissociation mechanism for NeIBr.

The energy gap law provides a means for qualitatively predicting the propensity for fragment rotational excitation $^{42}$ by calculating the quantity $\alpha\left(\mu_{t} / \mu_{r}\right)^{1 / 2}$, in which $\mu_{t}$ is the reduced mass of the rare gas-halogen complex, $\mu_{r}$ is the reduced mass of the diatomic halogen fragment, and

$$
\alpha \equiv \frac{m_{A}}{m_{A}+m_{B}} .
$$

(The diatomic halogen is represented as $A-B$, with $B$ being the nearest-neighbor to the rare gas atom.) Larger values of the quantity $\alpha\left(\mu_{t} / \mu_{r}\right)^{1 / 2}$ are associated with complexes in which larger degrees of rotational excitation are likely. For complexes containing homonuclear halogen diatomics, $\alpha=0.5$, while for $\mathrm{NeIBr}, \alpha=0.62$ ( $\mathrm{Br}$ is nearest-neighbor) and for NeICl, $\alpha=0.78$ ( $\mathrm{Cl}$ is nearest-neighbor). ${ }^{19} \alpha \neq 0.5$ denotes a complex with mass asymmetry in the diatomic fragment; $\alpha>0.5$ suggests a complex in which the rare gas atom is positioned, at equilibrium, closer to the light end of the diatom where it can provide the greatest degree of impulsive rotational excitation. In Table II, we list the calculated values of $\alpha\left(\mu_{t} / \mu_{r}\right)^{1 / 2}$ for a series of Ne-halogen complexes with similar amounts of available energy, along with the fraction of available energy that appears as fragment rotation. Note that the energy gap expression correctly predicts the order of importance of fragment rotation for complexes with quite different equilibrium structures, though not the magnitude of the variation in rotational excitation from one complex to another.

Sivakumar et al. have tested the ability of the energy gap concept to predict the form of the $\mathrm{Br}_{2}$ fragment rota-

TABLE II. Energy gap analysis of Ne-halogen complexes.

\begin{tabular}{|c|c|c|c|c|c|}
\hline Complex & $\alpha\left(\mu_{r} / \mu_{r}\right)^{1 / 2}$ & $E_{\mathrm{avl}}\left(\mathrm{cm}^{-1}\right)$ & $\left\langle E_{\mathrm{rot}}\right\rangle\left(\mathrm{cm}^{-1}\right)$ & $\left\langle E_{\mathrm{rot}}\right\rangle / E_{\text {avl }}$ & Reference \\
\hline $\mathrm{NeBr}_{2}$ & 0.34 & $70^{\mathrm{a}}$ & 5 & 0.07 & 18 \\
\hline \multirow[t]{2}{*}{$\mathrm{NeIBr}$} & 0.38 & $68.2^{\mathrm{b}}$ & 8.9 & 0.13 & this work \\
\hline & & $78.3^{\mathrm{c}}$ & 11.0 & 0.14 & this work \\
\hline \multirow[t]{2}{*}{$\mathrm{NeCl}_{2}$} & 0.47 & $66.3^{d}$ & 10.8 & 0.16 & 13 \\
\hline & & $77.2^{e}$ & 13.2 & 0.17 & 13 \\
\hline $\mathrm{NeICl}$ & 0.63 & $75^{f}$ & 15.0 & 0.20 & 9 \\
\hline
\end{tabular}

$v=10 \rightarrow v=9, B$ electronic state.

$b^{b} v 17 \rightarrow v=15, A$ electronic state.

$c_{v=16} \rightarrow v=14, A$ electronic state.

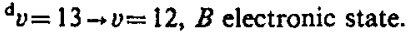

$e^{e}=14 \rightarrow v=13, B$ electronic state.

$\mathrm{f} v=14 \rightarrow v=13, A$ electronic state; the $D_{0}$ for $\mathrm{NeICl}$ is taken from Ref. 44 . 


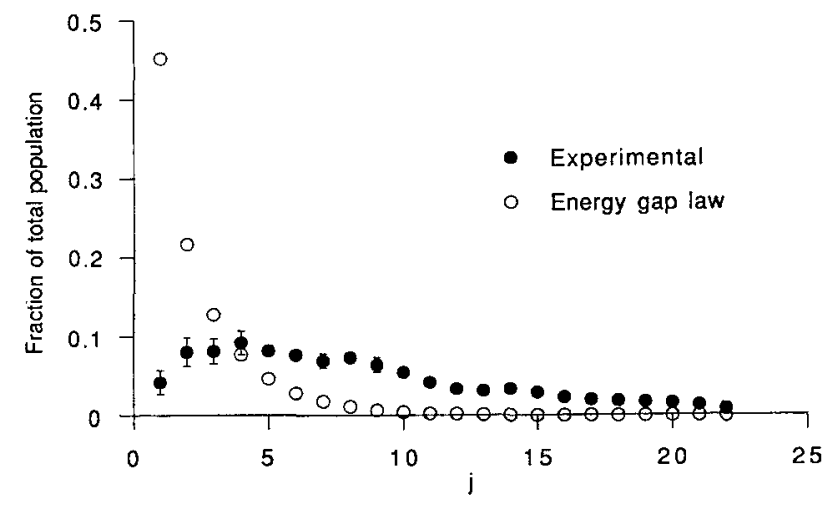

FIG. 9. Energy gap analysis of $\mathrm{IBr}$ fragment rotational excitation (open circles) compared with the experimental data (solid circles) for the $\Delta v$ $=-1$ dissociation of $v=12 \mathrm{NeIBr}$.

tional distribution from the dissociation of $\mathrm{NeBr}_{2} \cdot{ }^{18} \mathrm{We}$ have carried out a similar calculation for the $v=12 \rightarrow v=11$ dissociation of NeIBr; the results are shown in Fig. 9. As found by Sivakumar et al. ${ }^{18}$ the distribution predicted by the energy gap relation peaks at the lowest $j$ levels, followed by an approximate exponential decline with increasing $j$. This result arises from the strong propensity against large changes in the rotational quantum number. By $j=9$, for example, the predicted population has decreased to $<1 \%$ of the total. Clearly, the energy gap law provides a poor representation of the rotational distribution, but Table II suggests that the model does contain useful qualitative aspects that can be applied to systems such as the rare gas-halogen complexes. The quantitative aspects of the energy gap concept will fail when the dynamics are poorly represented by a direct Golden Rule coupling between the initial and final states (as in an IVR mechanism) ${ }^{4,5}$ or when final state interactions and not wave function overlap dominate the rotational excitation of the fragments. It is particularly interesting to note that the relatively large rotational excitation of the $\mathrm{ICl}$ fragments from the dissociation of $\mathrm{NeICl}$ is qualitatively predicted once the mass asymmetry factor $\alpha$ is incorporated. This result, which is based purely on kinematic considerations, suggests that classical trajectory calculations on this system may be particularly valuable, as the importance of final state interactions will be revealed. We anticipate applying the model of Waterland et al. ${ }^{10}$ to $\mathrm{NeICl}$ in the near future along with quantum dynamics calculations on NeIBr. Our goal is to use insights drawn from these calculations to develop a comprehensive framework for the disposal of energy in the dissociation of rare gas-halogen van der Waals molecules.

\section{ACKNOWLEDGMENTS}

The calculations described in this manuscript were carried out while the author was on sabbatical leave at the Department of Chemistry, University of Pennsylvania, where I was the beneficiary of numerous valuable discussions with Professor Marsha Lester. The hospitality of her research group is gratefully acknowledged. I am also grateful to Dr. Nadine Halberstadt for providing the software codes used to calculate the bound state energies and wave functions of $\mathrm{NeIBr}$ and for her assistance in their implementation. This research has been supported a Eugene M. Lang Faculty Fellowship from Swarthmore College and by a grant from the National Science Foundation (CHE8915038).

${ }^{1}$ See, for example, D. H. Levy, Adv. Chem. Phys. 47, 323 (1981); K. C. Janda, ibid. 60, 201 (1985); K. C. Janda and C. R. Bieler, in Atomic and Molecular Clusters, edited by E. R. Bernstein (Elsevier, Amsterdam, 1990), p. 455.

${ }^{2}$ J. A. Beswick and G. Delgado-Barrio, J. Chem. Phys. 73, 3653 (1980).

${ }^{3}$ N. Halberstadt, O. Roncero, and J. A. Beswick, Chem. Phys. 129, 83 (1989).

${ }^{4}$ N. Halberstadt, J. A. Beswick, O. Roncero, and K. C. Janda, J. Chem. Phys. 96, 2404 (1992).

${ }^{5}$ N. Halberstadt, S. Serna, O. Roncero, and K. C. Janda, J. Chem. Phys. 97, 341 (1992).

${ }^{6}$ J. C. Drobits, J. M. Skene, and M. I. Lester, J. Chem. Phys. 84, 2896 (1986).

${ }^{7}$ J. M. Skene, J. C. Drobits, and M. I. Lester, J. Chem. Phys. 85, 2329 (1986).

${ }^{8}$ J. C. Drobits and M. I. Lester, J. Chem. Phys. 88, 120 (1987).

${ }^{9}$ J. C. Drobits and M. I. Lester, J. Chem. Phys. 89, 4716 (1988).

${ }^{10}$ R. L. Waterland, J. M. Skene, and M. I. Lester, J. Chem. Phys. 89, 7277 (1988).

${ }^{11}$ J. I. Cline, B. P. Reid, D. D. Evard, N. Sivakumar, N. Halberstadt, and K. C. Janda, J. Chem. Phys. 89, 3535 (1988).

${ }^{12}$ W. D. Sands, C. R. Bieler, and K. C. Janda, J. Chem. Phys. 95, 729 (1991).

${ }^{13}$ J. I. Cline, N. Sivakumar, D. D. Evard, C. R. Bieler, B. P. Reid, N. Halberstadt, S. R. Hair, and K. C. Janda, J. Chem. Phys. 90, 2605 (1989).

${ }^{14}$ S. R. Hair, J. I. Cline, C. R. Bieler, and K. C. Janda, J. Chem. Phys. 90, 2935 (1989).

${ }^{15}$ D. D. Evard, C. R. Bieler, J. I. Cline, N. Sivakumar, and K. C. Janda, J. Chem. Phys. 89, 2829 (1988).

${ }^{16}$ C. R. Bieler, D. D. Evard, and K. C. Janda, J. Phys. Chem. 94, 7452 (1990).

${ }^{17}$ C. R. Bieler, K. E. Spence, and K. C. Janda, J. Phys. Chem. 95, 5058 (1991).

${ }^{18}$ N. Sivakumar, J. I. Cline, C. R. Bieler, and K. C. Janda, Chem. Phys. Lett. 147, 561 (1988)

${ }^{19}$ O. Roncero, J. A. Beswick, N. Halberstadt, P. Villarreal, and G. Delgado-Barrio, J. Chem. Phys. 92, 3348 (1990).

${ }^{20}$ N. Halberstadt, J. A. Beswick, and K. C. Janda, J. Chem. Phys. 87, 3966 (1987).

${ }^{21}$ R. L. Waterland, M. I. Lester, and N. Halberstadt, J. Chem. Phys. 92, 4261 (1990).

${ }^{22}$ B. P. Reid, K. C. Janda, and N. Halberstadt, J. Phys. Chem. 92, 587 (1988).

${ }^{23}$ S. K. Gray and C. E. Wozny, J. Chem. Phys. 94, 2817 (1991).

${ }^{24}$ R. Schinke, Annu. Rev. Phys. Chem. 39, 39 (1988).

${ }^{25}$ R. Schinke, J. Chem. Phys. 85, 5049 (1986).

${ }^{26}$ S. A. Walter and T. A. Stephenson, J. Chem. Phys. 96, 3536 (1992).

${ }^{27}$ Calculated using the programs RKR1 and LEVEL by R. J. LeRoy (University of Waterloo Chemical Physics Research Report, No. CP-230R3, 1986).

${ }^{28}$ M. Suzuki and T. Fujiwara, J. Mol. Spectrose. 133, 233 (1989).

${ }^{29} \mathrm{~B}$. Guo and J. Tellinghuisen, J. Mol. Spectrosc. 127, 222 (1988).

${ }^{30}$ J. C. D. Brand, D. R. Dhatt, A. R. Hoy, and D. C. P. Tse, J. Mol. Spectrosc. 119, 398 (1986).

${ }^{31}$ W. R. Simpson and T. A. Stephenson, J. Chem. Phys. 90, 3171 (1989).

${ }^{32}$ See, for example, R. Vasudev, R. Zare, and R. N. Dixon, J. Chem. Phys. 80, 4863 (1984); P. Andresen, G. S. Ondrey, B. Titze, and E. Rothe, ibid. 80, 2548 (1984).

${ }^{33}$ C. H. Greene and R. N. Zare, J. Chem. Phys. 78, 6741 (1983).

${ }^{34}$ A. J. Sadlej, J. Chem. Phys. 96, 2048 (1992).

${ }^{39}$ Handbook of Chemistry and Physics, 67th ed. (Chemical Rubber, Columbus, 1986), p. E-68.

${ }^{36}$ The band shift for the $(16,0) A-X$ transition in $\mathrm{NeIBr}$ is $6.3 \pm 0.2$ $\mathrm{cm}^{-1}$. This value was omitted from Ref. 31 . 
${ }^{37}$ In all of our calculations, we assume that the electronic angular momentum of the $\operatorname{IBr} A(\Omega=1)$ electronic state is a spectator to the dissociation dynamics and is not considered. When we compare experimental and theoretical results, we correlate the calculated $j=0$ population with the observed $j=1$ population, the calculated $j=1$ population with the observed $j=2$ population, and so forth.

${ }^{38}$ M. D. Morse, K. F. Freed, and Y. B. Band, J. Chem. Phys. 70, 3604 (1979); M. D. Morse and K. F. Freed, ibid. 78, 6045 (1983).

${ }^{39}$ R. Schinke, Chem. Phys. Lett. 120, 129 (1985).
${ }^{40}$ D. W. Noid, S. K. Gray, and S. A. Rice, J. Chem. Phys. 84, 2649 (1986).

${ }^{41}$ J. A. Beswick and J. Jortner, Adv. Chem. Phys. 47, 363 (1981).

${ }^{42}$ G. E. Ewing, J. Phys. Chem. 91, 4662 (1987).

${ }^{43}$ B. A. Swartz, D. E. Brinza, C. M. Western, and K. C. Janda, J. Phys. Chem. 88, 6272 (1984).

${ }^{44}$ T. A. Stephenson, Y. Hong, and M. I. Lester, J. Chem. Phys. 94, 4171 (1991). 